\title{
Fluorine in the solar neighborhood: Chemical evolution models
}

\author{
E. Spitoni ${ }^{1,2}$, F. Matteucci ${ }^{1,2,3}$, H. Jönsson ${ }^{4}$, N. Ryde ${ }^{4}$, and D. Romano ${ }^{5}$ \\ ${ }^{1}$ Dipartimento di Fisica, Sezione di Astronomia, Università di Trieste, Via G.B. Tiepolo 11, 34131 Trieste, Italy \\ e-mail: spitoni@oats.inaf.it \\ 2 INAF - Osservatorio Astronomico di Trieste, Via G.B. Tiepolo 11, 34131 Trieste, Italy \\ ${ }^{3}$ INFN - Sezione di Trieste, Via Valerio 2, 34100 Trieste, Italy \\ ${ }^{4}$ Lund Observatory, Department of Astronomy and Theoretical Physics, Lund University, Box 43, 22100 Lund, Sweden \\ 5 INAF - Osservatorio Astronomico di Bologna, Via Gobetti 93/3, 40129 Bologna, Italy
}

Received 12 October 2017 / Accepted 25 November 2017

\begin{abstract}
Context. In light of new observational data related to fluorine abundances in solar neighborhood stars, we present chemical evolution models testing various fluorine nucleosynthesis prescriptions with the aim to best fit those new data.

Aims. We consider chemical evolution models in the solar neighborhood testing various nucleosynthesis prescriptions for fluorine production with the aim of reproducing the observed abundance ratios $[\mathrm{F} / \mathrm{O}]$ versus $[\mathrm{O} / \mathrm{H}]$ and $[\mathrm{F} / \mathrm{Fe}]$ versus $[\mathrm{Fe} / \mathrm{H}]$. We study in detail the effects of various stellar yields on fluorine production.

Methods. We adopted two chemical evolution models: the classical two-infall model, which follows the chemical evolution of halothick disk and thin disk phases; and the one-infall model, which is designed only for thin disk evolution. We tested the effects on the predicted fluorine abundance ratios of various nucleosynthesis yield sources, that is, asymptotic giant branch (AGB) stars, Wolf-Rayet (W-R) stars, Type II and Type Ia supernovae, and novae.

Results. The fluorine production is dominated by AGB stars but the W-R stars are required to reproduce the trend of the observed data in the solar neighborhood with our chemical evolution models. In particular, the best model both for the two-infall and one-infall cases requires an increase by a factor of 2 of the W-R yields. We also show that the novae, even if their yields are still uncertain, could help to better reproduce the secondary behavior of $\mathrm{F}$ in the $[\mathrm{F} / \mathrm{O}]$ versus $[\mathrm{O} / \mathrm{H}]$ relation.

Conclusions. The inclusion of the fluorine production by W-R stars seems to be essential to reproduce the new observed ratio [F/O] versus $[\mathrm{O} / \mathrm{H}]$ in the solar neighborhood. Moreover, the inclusion of novae helps to reproduce the observed fluorine secondary behavior substantially.
\end{abstract}

Key words. Galaxy: abundances - Galaxy: evolution - ISM: general

\section{Introduction}

The aim of this paper is to study the evolution of fluorine $\left({ }^{19} \mathrm{~F}\right)$ abundance in the solar neighborhood by means of a detailed chemical evolution model. The origin of fluorine is still uncertain and widely debated in literature. The production of the only stable isotope, ${ }^{19} \mathrm{~F}$, is strictly linked to the physical conditions in stars. In particular, we can identify several stellar sites for fluorine production:

(i) Asymptotic giant branch (AGB) stars. For solar metallicity the main process of ${ }^{19} \mathrm{~F}$ production is related to nuclear reaction on ${ }^{14} \mathrm{~N}$ including $\alpha$, neutron, and proton captures. On the other hand, at lower metallicity the ${ }^{19} \mathrm{~F}$ production depends on ${ }^{13} \mathrm{C}$ (Cristallo et al. 2014).

(ii) Wolf-Rayet (W-R) stars can be important producers of fluorine, which is injected into the interstellar medium (ISM) by their strong stellar winds. Also, the chain of reactions leading to ${ }^{19} \mathrm{~F}$ production starts from ${ }^{14} \mathrm{~N}$, which is normally produced during the $\mathrm{CNO}$ cycle as a secondary element. However, in massive stars $\mathrm{N}$ can be produced as a primary element if they suffer strong rotation, which produces effects similar to those of the dredge-up mechanism in AGB stars. In this case, however, the primary $\mathrm{N}$ production is limited only to very metal poor stars $\left(Z<10^{-5}\right)$ as shown by Meynet \& Maeder (2002). The difference in secondary and primary $\mathrm{N}$ is important since the $\mathrm{F}$ behavior would also follow that of a secondary element in the case of secondary $\mathrm{N}$, namely depending on the original stellar metallicity.

(iii) Type II supernovae (SNe II). These $\mathrm{SNe}$ can produce ${ }^{19} \mathrm{~F}$ via the neutrino $(v)$ process. In fact, although neutrinos are characterized by small cross sections, the great amount of neutrinos released during the core-collapse turns ${ }^{20} \mathrm{Ne}$, in the outer envelopes of the collapsing star, into ${ }^{19} \mathrm{~F}$ (Woosley \& Haxton 1988).

(iv) Novae can also in principle be ${ }^{19} \mathrm{~F}$ producers (José \& Hernanz 1998), although the yields are still uncertain. In classical novae the reaction chain ${ }^{17} \mathrm{O}(\mathrm{p}, \gamma){ }^{18} \mathrm{~F}(\mathrm{p}, \gamma){ }^{19} \mathrm{Ne}$ is the mechanism involved in the synthesis of ${ }^{19} \mathrm{~F}$ through the production of the short-lived, $\beta^{+}$-unstable nucleus ${ }^{19} \mathrm{Ne}$, which is partially transferred by convection toward the outer cooler layers of the envelope, where it decays into ${ }^{19} \mathrm{~F}$.

Recently, new observational data (related to $49 \mathrm{~K}$ giants with temperatures low enough to show an HF line at $2.3 \mu \mathrm{m}$ ) by Jönsson et al. (2017a) showed that the $v$ process is not the main contributor of fluorine production in the solar vicinity, at variance with theoretical predictions. Jönsson et al. (2017b) estimated the kinematic probability that stars belong to the thin disk, thick disk, and/or halo. These authors showed that the majority of the observed stars are part of the Galactic thin disk. 
The observed $[\mathrm{F} / \mathrm{Fe}]$ versus $[\mathrm{Fe} / \mathrm{H}]$ and $[\mathrm{F} / \mathrm{O}]$ versus $[\mathrm{O} / \mathrm{H}]$ ratio both follow an increasing trends that is in contrast to what would be expected if the $v$ process was the dominant fluorine nucleosynthesis source in the solar vicinity, constraining its possible contribution to the cosmic fluorine budget. This tension between data and the $v$ process in the solar neighborhood is confirmed by the observed secondary relation between fluorine and oxygen in Jönsson et al. (2017a). This observed secondary behavior can constrain the stellar models of AGB and W-R stars. Hence, AGB stars, W-R stars, SNe Ia, novae, and explosive nucleosynthesis in SNe II without $v$ process are possible fluorine production sites to be tested in detailed chemical evolution models of the solar neighborhood.

In this paper, we present chemical evolution models designed to reproduce the data by Jönsson et al. (2017a) in the solar neighborhood testing various nucleosynthesis prescriptions for the fluorine yields. We present results related both to an updated version of the classical two-infall model introduced by Chiappini et al. (1997) in which we follow the evolution of the halo-thick disk and thin disk phases, and to a detailed chemical evolution model that follows the thin disk evolution alone. The $\mathrm{F}$ production sources considered in this paper are AGB stars (Karakas 2010), massive stars (Kobayashi et al. 2006), and W-R stars (Meynet \& Arnould 2000). We also discuss the case of novae as possible sources for $\mathrm{F}$ production adopting the nucleosynthesis yields by José \& Hernanz (1998).

Previous papers (Meynet \& Arnould 2000; Renda et al. 2004; Kobayashi et al. 2011a) have computed fluorine evolution in the Galaxy. For the first time, Meynet \& Arnould (2000) showed that W-R stars could be significant contributors to the solar system abundance of fluorine using a simple model for chemical evolution. Renda et al. (2004) considered the impact of fluorine nucleosynthesis in both W-R and AGB stars using a chemical evolution model and these authors concluded that the contribution of W-R stars is necessary to reproduce the F abundance in solar vicinity. Kobayashi et al. (2011a) found that the main effect of $v$ process of core-collapse supernovae on the evolution of fluorine in the solar neighborhood is the presence of a plateau at high $[\mathrm{F} / \mathrm{O}]$ values for $[\mathrm{O} / \mathrm{H}]<-1.2$ in the $[\mathrm{F} / \mathrm{O}]$ versus $[\mathrm{O} / \mathrm{H}]$ relation. As underlined above, this plateau is in contrast with the most recent observational data by Jönsson et al. (2017a). Novae have so far never been included in chemical models predicting fluorine.

The paper is organized as follows: in Sect. 2 we describe the chemical evolution models for the solar vicinity adopted in this paper; in Sect. 3 the nucleosynthesis prescriptions are described. In Sect. 4 our results concerning the fluorine abundances in the solar neighborhood predicted by our chemical evolution models are reported. In Sect. 5, we discuss the effects of the novae nucleosynthesis on fluorine production. In Sect. 6 we draw our main conclusions.

\section{Chemical evolution models for the solar neighborhood}

In this section, we describe the main characteristics of the adopted chemical evolution models for the solar neighborhood in this work. First, in the classical two-infall model of Chiappini et al. (1997, 2001), it is assumed that the Galaxy formed with two independent infalls of primordial gas. In the first episode, which occurred on short timescales, the halo-thick disk components were formed, while in the second infall the thin disk was created on longer timescales. Second, following the
Matteucci \& François (1989) approach, we used the one-infall chemical evolution model only for the thin disk evolution in the solar neighborhood.

\subsection{The two-infall model}

As stated in Sect. 2, Jönsson et al. (2017a) data sample is related to thick disk, thin disk and halo stars. For this reason, first we want to reproduce those data adopting the classical two-infall model that is capable of tracing the chemical evolution of halothick disk and thin disk phases.

The Galaxy is assumed to have formed by means of two main infall episodes. The accretion law for a certain element $i$ at the time $t$ in the solar vicinity is defined as

$$
\mathcal{A}(t, i)=X_{A_{i}}\left[c_{1} \mathrm{e}^{-t / \tau_{\mathrm{H}}}+c_{2} \mathrm{e}^{-\left(t-t_{\max }\right) / \tau_{\triangleright}}\right] .
$$

The quantity $X_{A_{i}}$ is the abundance by mass of the element $i$ in the infalling gas, while $t_{\max }=1 \mathrm{Gyr}$ is the time for the maximum infall on the thin disk, $\tau_{\mathrm{H}}=0.8 \mathrm{Gyr}$ is the timescale for the creation of the halo and thick disk and $\tau_{\mathrm{D}}=7 \mathrm{Gyr}$ is the timescale to build up the thin disk in the solar neighborhood (as suggested by fitting the G-dwarf metallicity distribution). Here, we assume that the abundances $X_{A_{i}}$ show primordial gas compositions. Finally, the coefficients $c_{1}$ and $c_{2}$ are obtained by imposing a fit to the observed current total surface mass density in the solar neighborhood. A threshold gas density of $7 M_{\odot} \mathrm{pc}^{-2}$ in the star formation process (Kennicutt 1989, 1998; Martin \& Kennicutt 2001) is also adopted for the disk.

The most recent observational data by the Gaia-ESO Survey (Recio-Blanco et al. 2014; Rojas-Arriagada et al. 2017), APOGEE (Hayden et al. 2015), and AMBRE (Mikolaitis et al. 2017) confirmed the existence of two distinct sequences corresponding to thick and thin disk stars. In Grisoni et al. (2017) it was shown that the two-infall model is able to reproduce perfectly the AMBRE data in the solar neighborhood when applied to thick and thin disks without including the halo.

\subsection{One-infall model}

Because the majority of the data presented in Jönsson et al. (2017a) are supposed to be thin disk stars, we also considered a chemical evolution model that only follows the thin disk.

To reproduce the chemical evolution of the thin disk, we adopted an updated version of the one-infall chemical evolution model presented by Matteucci \& François (1989) using the most recent nucleosynthesis yield of Romano et al. (2010).

The infall rate in the thin disk for a given element $i$ at the time $t$, and in the solar vicinity is defined as

$\mathcal{B}(t, i)=X_{A_{i}} c_{2} \mathrm{e}^{-\frac{t}{\tau_{\mathrm{D}}}}$.

All model parameters are the same as the thin disk phase of the two-infall model described in Sect. 2.1.

\section{Nucleosynthesis prescriptions for $\mathrm{O}, \mathrm{Fe}$, and $\mathrm{F}$}

In this work our principal aim is to analyze in detail the contribution of the different channels that produce fluorine in the solar neighborhood by comparing the predicted abundance ratios $[\mathrm{F} / \mathrm{Fe}]$ versus $[\mathrm{Fe} / \mathrm{H}]$ and $[\mathrm{F} / \mathrm{O}]$ versus $[\mathrm{O} / \mathrm{H}]$ of our chemical evolution models with the data.

We start our study by considering the set of nucleosynthesis yields of the Romano et al. (2010) best model (their model 15) 
Table 1. List of the models described in this work in which we considered different contributions of fluorine production.

\begin{tabular}{ccccc}
\hline \hline Models & $\begin{array}{c}\text { AGB stars } \\
\text { Karakas (2010) }\end{array}$ & $\begin{array}{c}\text { SNe Ia } \\
\text { Iwamoto et al. (1999) }\end{array}$ & $\begin{array}{c}\text { SNe II } \\
\text { Kobayashi et al. (2006) }\end{array}$ & $\begin{array}{c}\text { Wolf-Rayet stars } \\
\text { Meynet \& Arnould (2000) }\end{array}$ \\
\hline F1 & Yes & Yes & Yes & No \\
F2 & No & Yes & Yes & No \\
F3 & Yes $\times 1.5$ & Yes & Yes & No \\
F4 & Yes $\times 2$ & Yes & Yes & No \\
F5 & Yes $\times 3$ & Yes & Yes & Yes \\
F6 & No & No & No & Yes \\
F7 & Yes & Yes & Yes & Yes $\times 2$ \\
F8 & Yes & Yes & Yes & Yes \\
F9 & Yes $\times 2$ & Yes & Yes & \\
\hline
\end{tabular}

Notes. In this table we report only the various nucleosynthesis yields adopted for the fluorine.

for $\mathrm{O}, \mathrm{Fe}$, and $\mathrm{F}$ (this set of yields has been adopted by Brusadin et al. 2013; Micali et al. 2013; Spitoni et al. 2016).

In particular, these yields are as follows:

- For low-and intermediate-mass stars $\left(0.8-8 \quad M_{\odot}\right)$, we considered the metallicity dependent stellar yields of Karakas (2010) with thermal pulses. These stars contribute to fluorine, negligibly to $\mathrm{O}$, and do not contribute to $\mathrm{Fe}$.

- For SNe Ia, the adopted nucleosynthesis prescriptions are from Iwamoto et al. (1999). These SNe contribute significantly to $\mathrm{Fe}$ and negligibly to $\mathrm{O}$ and $\mathrm{F}$.

- For massive stars $\left(M>8 M_{\odot}\right)$, which are the progenitors of either SNe II or hypernovae $(\mathrm{HNe})$, depending on the explosion energy, we assumed the metallicity dependent $\mathrm{He}, \mathrm{C}$, $\mathrm{N}$, and $\mathrm{O}$ stellar yields, as computed with the Geneva stellar evolutionary code, which takes into account the combined effect of mass loss and rotation (Meynet \& Maeder 2002; Hirschi 2005, 2007; Ekström et al. 2008). Kobayashi et al. (2006) yields not including $v$ nucleosynthesis were considered for fluorine. Jönsson et al. (2017a) compared their new observational data with chemical evolution models by Kobayashi et al. (2011b) that included $v$ nucleosynthesis. This model showed a plateau at high $[\mathrm{F} / \mathrm{O}]$ values and at low $[\mathrm{O} / \mathrm{H}]$ in contrast with the new data. For this reason we did not take into account this kind of nucleosynthesis in this work.

In this paper we also test the effects of W-R yields. For W-R stars we assumed the F production by the models with mass loss by Meynet \& Arnould (2000). These yields do not include oxygen.

Meynet \& Arnould (1993) have suggested that W-R stars could significantly contaminate the Galaxy with ${ }^{19} \mathrm{~F}$. In their scenario, ${ }^{19} \mathrm{~F}$ is synthesized at the beginning of the He-burning phase from the ${ }^{14} \mathrm{~N}$ left over by the previous CNO-burning core, and is ejected in the ISM when the star enters its WC phase. Since the mass loss depends on stellar metallicity, the ${ }^{19} \mathrm{~F}$ yields are metallicity dependent.

For all the elements heavier than oxygen, namely $\mathrm{Fe}$ in this study, we assumed the Kobayashi et al. (2006) yields.

\section{Results: testing various nucleosynthesis prescriptions for fluorine}

In this section, we show the results related to various prescriptions for fluorine yields with the aim of reproducing the observational data by Jönsson et al. (2017a) both for the twoinfall model and for a model that only includes a study of the

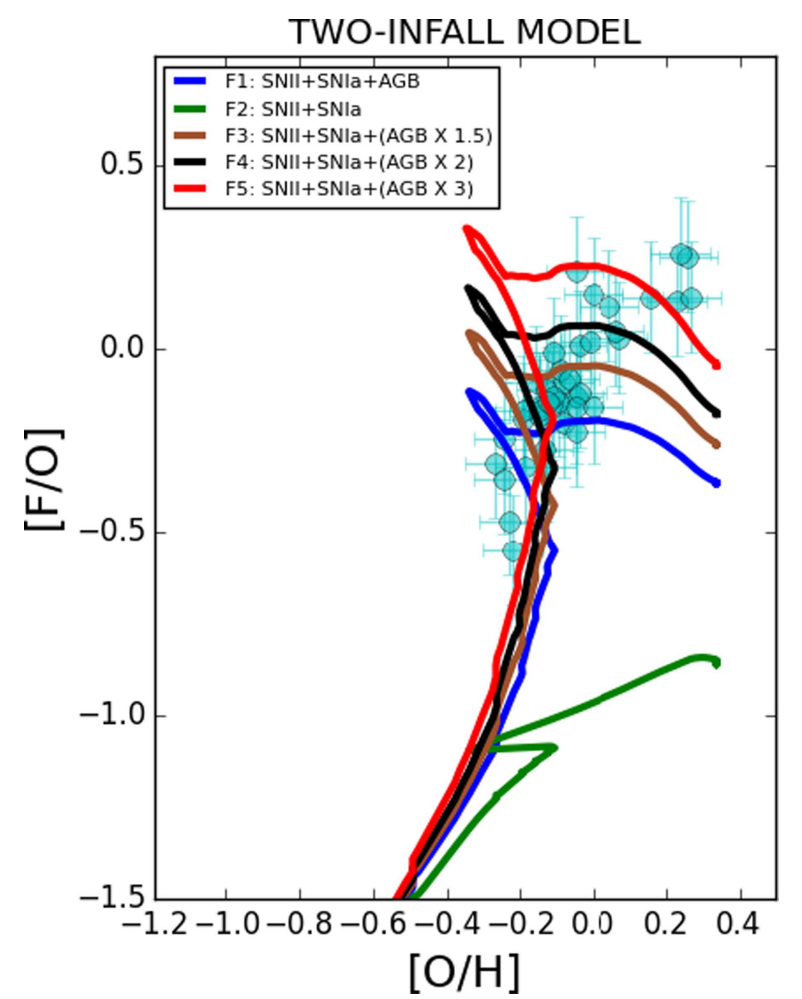

Fig. 1. Abundance ratio $[\mathrm{F} / \mathrm{O}]$ as a function of $[\mathrm{O} / \mathrm{H}]$ in the solar neighborhood for the two-infall chemical evolution model adopting various prescriptions for the channels of the fluorine production. For oxygen we use the Romano et al. (2010; model 15) prescriptions. The blue line represents the model F1 of Table 2, where fluorine is assumed to be produced by both AGB stars, SNe Ia, and SNe II. The green line indicates the model F2 (contributions to fluorine production only from SNe Ia and SNe II). The models F3, F4, and F5 in which the AGB yields are multiplied by factors of $1.5,2$, and 3, respectively (along with SN Ia, SN II channels) are indicated with brown, black, and red lines. Observational data by Jönsson et al. (2017a) are indicated with cyan circles.

evolution of the thin disk (one-infall model). In Table 1 we present the list of all models tested, varying the fluorine yield prescriptions. For each model $(\mathrm{F} 1, \ldots, \mathrm{F} 9)$, we indicate the presence of AGB stars with yields by Karakas (2010) in col. 2, Type Ia SNe with yields by Iwamoto et al. (1999) in col. 3, massive stars with yields by Kobayashi et al. (2006) in col. 4, and W-R stars with yields by Meynet \& Arnould (2000) in col. 5. As stated above we started our work analyzing the effects of the 


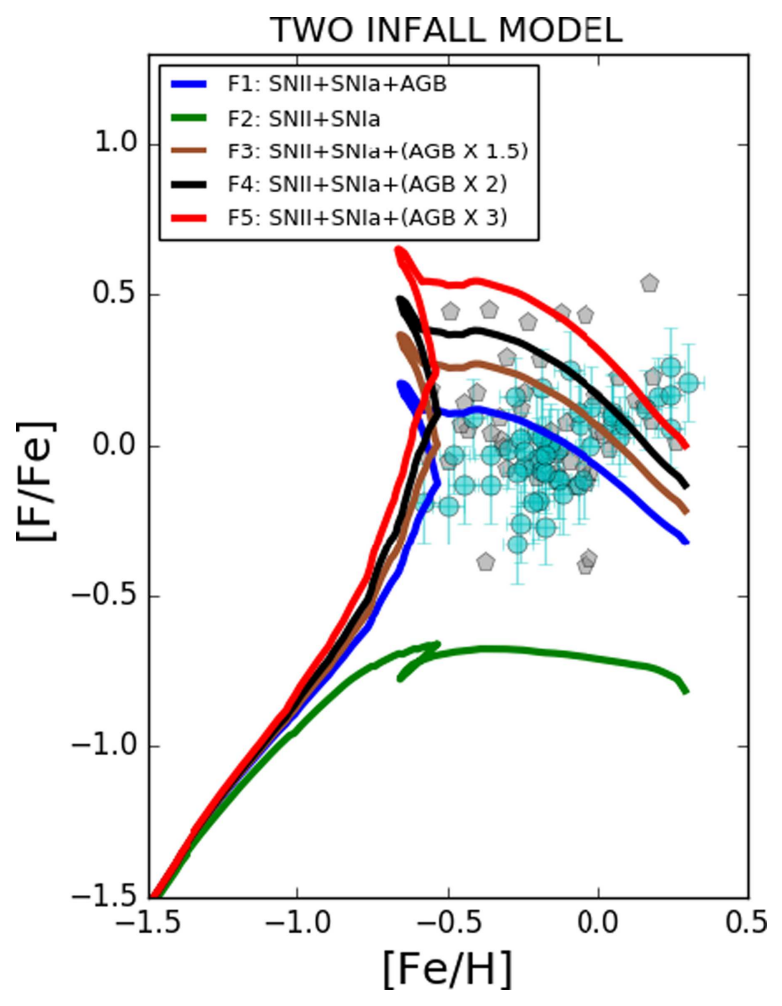

Fig. 2. Abundance ratio $[\mathrm{F} / \mathrm{Fe}]$ as a function of $[\mathrm{Fe} / \mathrm{H}]$ in the solar neighborhood for the two-infall chemical evolution model adopting various prescriptions for the channels of fluorine production. Model lines are those described in Fig. 1. Observational data of Jönsson et al. (2017a) are indicated with cyan circles, whereas the data taken by Pilachowski $\&$ Pace (2015) are presented with gray pentagons.

nucleosynthesis prescriptions of the best model by Romano et al. (2010; their model 15 ) on the $[\mathrm{F} / \mathrm{O}]$ versus $[\mathrm{O} / \mathrm{H}]$ and $[\mathrm{F} / \mathrm{Fe}]$ versus $[\mathrm{Fe} / \mathrm{H}]$ ratios in the light of the new data by Jönsson et al. (2017a). We label this model as F1.

The solar values adopted in this work for oxygen and iron are from Asplund et al. (2009), whereas the fluorine value computed by Maiorca et al. (2014) is used to be coherent with Jönsson et al. (2017a).

We stress that in this work we analyze in detail only fluorine yields; for the other elements shown in this paper $(\mathrm{O}, \mathrm{Fe})$, we used the model 15 prescriptions by Romano et al. (2010), which does not include fluorine, however.

\subsection{Two-infall model results}

Jönsson et al. (2017a) compared their new observational data with chemical evolution models by Kobayashi et al. (2011a,b) for thick and thin disk stars; the latter work considered the contribution of AGB stars and $v$ processes to fluorine production. The main problem of those models is that they are not capable of reproducing the slope of the secondary behavior of the new data presented by Jönsson et al. (2017a).

Here, we show the results related to the two-infall model of Chiappini et al. (1997, 2001) updated by Romano et al. (2010) for the chemical evolution of the solar neighborhood. In Fig. 1 we show the abundance ratio $[\mathrm{F} / \mathrm{O}]$ as a function of $[\mathrm{O} / \mathrm{H}]$ in the solar neighborhood adopting various prescriptions for the channels of fluorine production. First, we consider, for $\mathrm{F}$ and $\mathrm{O}$, the nucleosynthesis yields adopted by the model 15 of Romano et al. (2010) (model F1 in Table 1), those of Karakas (2010) for AGB stars with thermal pulses, and those of Iwamoto et al. (1999)
TWO INFALL MODEL

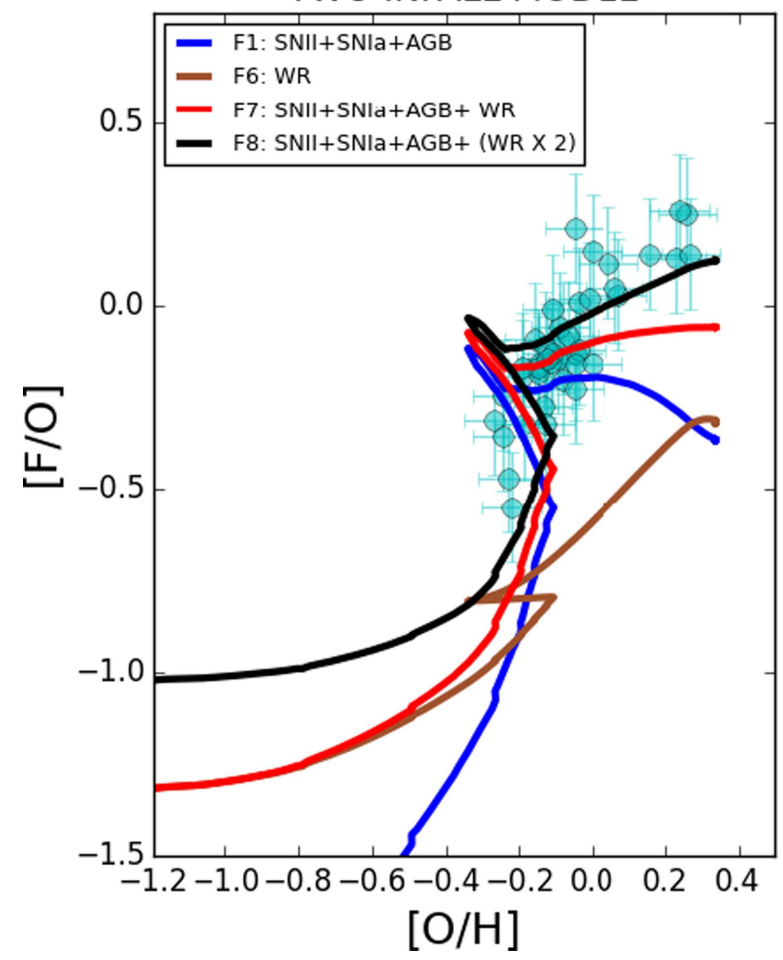

Fig. 3. Abundance ratio $[\mathrm{F} / \mathrm{O}]$ as a function of $[\mathrm{O} / \mathrm{H}]$ in the solar neighborhood for the two-infall chemical evolution model testing different prescriptions for the channels of fluorine production. With the blue line we represent the model F1 of Table 1. With the red line we consider also the W-R yield by Meynet \& Arnould (2000). Model F6 where only W-R stars contribute to the fluorine production is indicated with the brown line in this plot. The black line is the model F8, similar to the F7 model but with the W-R yields multiplied by a factor of 2 . Observational data of Jönsson et al. (2017a) are indicated with cyan circles.

for Type Ia SNe. We also consider the nucleosynthesis yields adopted by Kobayashi et al. (2006) for massive stars, for F, and the Geneva stellar evolutionary code, for $\mathrm{O}$.

In Fig. 1, model F1 clearly shows the transition between the halo-thick disk phase and the thin disk. Because of the presence of a threshold for the surface gas density in the star formation we have a gap in the star formation history (see Spitoni et al. 2016) in correspondence with the beginning of the second gas infall.

At variance with the models of Kobayashi et al. (2011a,b) shown in Fig. 3 of Jönsson et al. (2017a), the F1 model shows a decrease of $[\mathrm{F} / \mathrm{O}]$ abundance values for $[\mathrm{O} / \mathrm{H}]$ larger than 0.2 dex. This behavior is due to the various prescriptions for the yield of oxygen we adopted. In fact, in Romano et al. (2010) the oxygen produced by massive stars is coming from the Geneva tracks with mass loss and rotation (Meynet \& Maeder 2002; Hirschi 2005, 2007; Ekström et al. 2008). Model F1 is not able to reproduce the observational trend of Jönsson et al. (2017a) data for over-solar values of $[\mathrm{O} / \mathrm{H}]$, and the main problem is that we are not capable of reaching the high $[\mathrm{F} / \mathrm{O}]$ observed values.

Figure 1 also shows that by adopting the Romano et al. (2010) best choice for the yields (their model 15), the main contribution to the fluorine is given by AGB stars. In fact, model F2 (see Table 1), in which the fluorine is created only by means of SN Ia and SN II channels, shows a very small amount of fluorine production: the $[\mathrm{F} / \mathrm{O}]$ abundance at maximum reaches the value of -0.8 dex.

However, the AGB yields of $\mathrm{F}$ are still uncertain. In fact, the recent measurements by Indelicato et al. (2017) and 


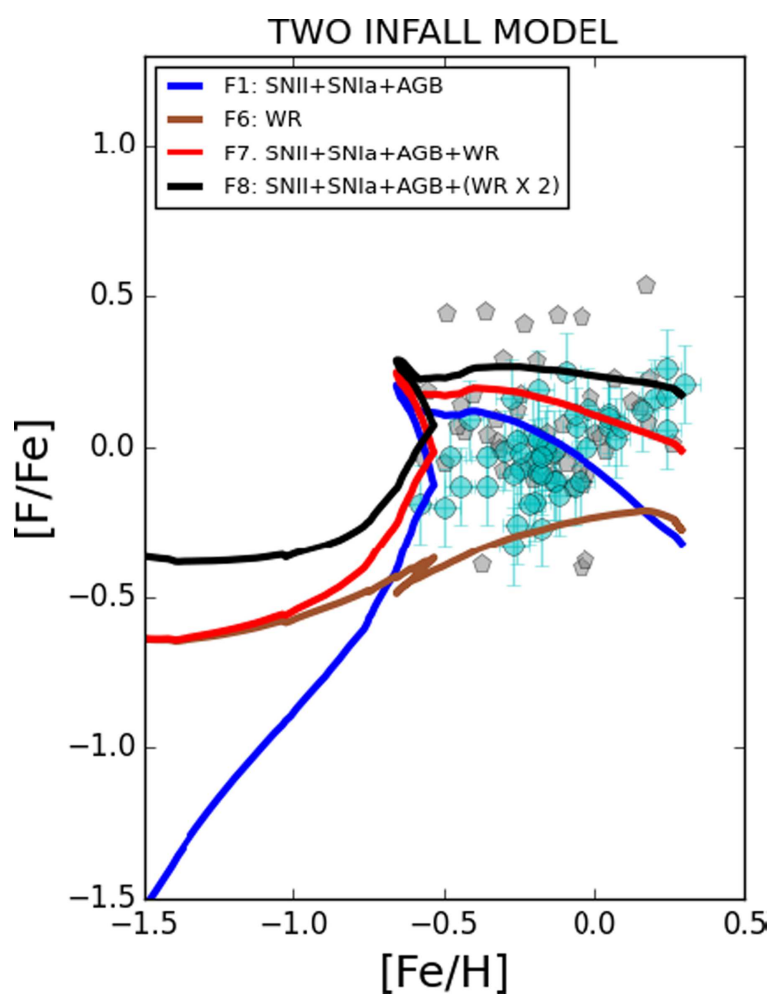

Fig. 4. Abundance ratio $[\mathrm{F} / \mathrm{Fe}]$ as a function of $[\mathrm{Fe} / \mathrm{H}]$ in the solar neighborhood for the two-infall chemical evolution model adopting different fluorine yields. Model lines are the ones described in Fig. 3. Observational data of Jönsson et al. (2017a) are indicated with cyan circles whereas the data taken by Pilachowski \& Pace (2015) are presented with gray pentagons.

He et al. (2018) of the ${ }^{19} \mathrm{~F}(\mathrm{p}, \alpha){ }^{16} \mathrm{O}$ reaction suggest that the computed yields from AGB stars should be revised and higher values might be expected. Therefore, in Fig. 1 the effects of increasing by hand the contribution by AGB stars are presented. Multiplying the yields by factors of $1.5,2$, and 3 (models F3, F4, and F5 in Table 1, respectively), we have a better agreement with the data.

This kind of procedure has been widely used in chemical evolution models in the past. For example, in the François et al. (2004) best model, the Mg yields related to the massive stars of Woosley \& Weaver (1995) have been multiplied by a factor of 2. More recently, in Maas et al. (2017) phosphorus yields by Kobayashi et al. (2006) were multiplied by a factor of 2.75 with the aim of reproducing the $[\mathrm{P} / \mathrm{Fe}]$ versus $[\mathrm{Fe} / \mathrm{H}]$ abundance ratios in the solar neighborhood as suggested by the new observational stellar data using the chemical evolution model by Cescutti et al. (2012). From Fig. 1 we see that models F4 and F5 are able to reach the observed high $[\mathrm{F} / \mathrm{O}]$ ratio values for oversolar $[\mathrm{O} / \mathrm{H}]$ abundances and trace the observed secondary slope of the data for subsolar $[\mathrm{O} / \mathrm{H}]$ values.

The data in Fig. 1 present a change in the slope of the [F/O] versus $[\mathrm{O} / \mathrm{H}]$ abundance ratios for values higher than $[\mathrm{O} / \mathrm{H}]=0$. The secondary behavior of the fluorine is not perfectly reproduced for over-solar values of $[\mathrm{O} / \mathrm{H}]$; in fact models $\mathrm{F} 3$ and $\mathrm{F} 4$ show a decrease in the $[\mathrm{F} / \mathrm{O}]$ abundance ratio for $[\mathrm{O} / \mathrm{H}]>0$.

A common problem of models F3, F4, and F5, is that the transition between the halo-thick disk phase and the thin disk has a big impact on the $[\mathrm{F} / \mathrm{O}]$ versus $[\mathrm{O} / \mathrm{H}]$ ratios when we adopted the Romano et al. (2010) model 15 set of yields. We show in the next subsection, concerning the one-infall results (the chemical

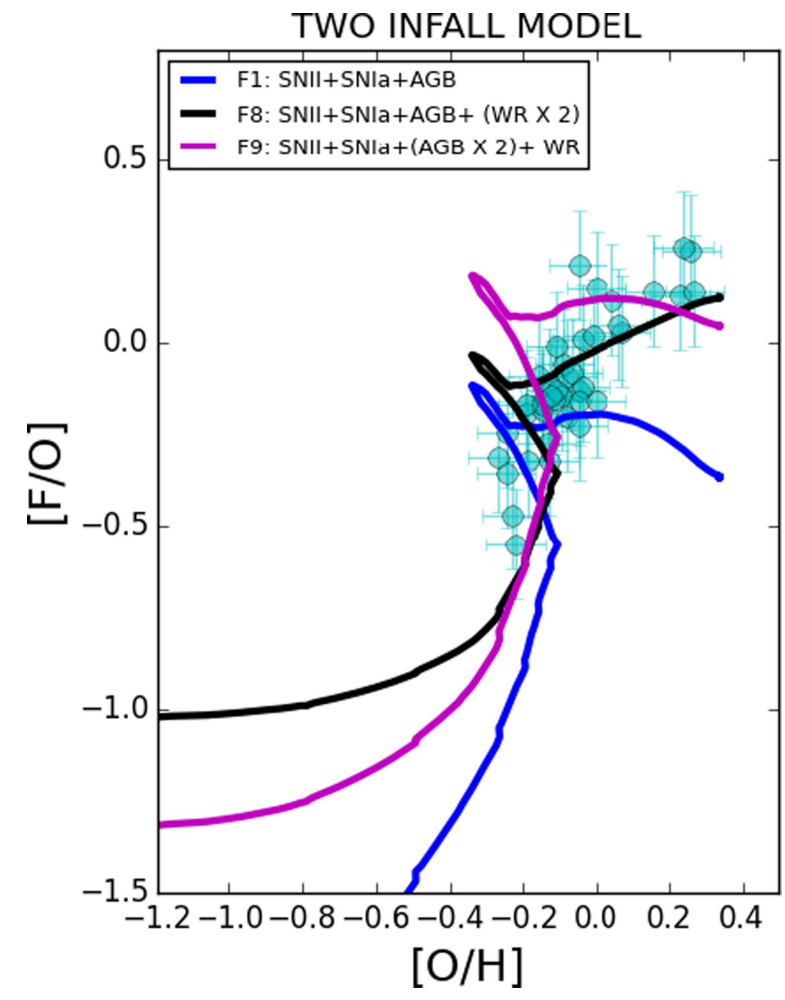

Fig. 5. Abundance ratio $[\mathrm{F} / \mathrm{O}]$ as a function of $[\mathrm{O} / \mathrm{H}]$ in the solar neighborhood for the two-infall chemical evolution model. The blue line represents the model $\mathrm{F} 1$ of Table 1 in which fluorine is assumed to be produced by both AGB stars and SNe Ia and SNe II. The model similar to the F7 model but with the W-R yields multiplied by a factor of 2 is indicated with the black line. The magenta line represents the model F9 in which the contribution of SNe II and SNe Ia are considered along with W-R stars. In this model the AGB yields are multiplied by a factor of 2. Observational data of Jönsson et al. (2017a) are indicated with cyan circles.

evolution only for the thin disk), that a better agreement with the data is achieved. We recall here that the majority of the data presented by Jönsson et al. (2017a) are parts of the thin disk system.

However, the predicted fluorine solar values are in agreement with solar values of Maiorca et al. (2014) as shown in Table 2. In fact, the predicted solar mass fraction for fluorine by the model F4 (AGB yields multiplied by a factor of 2 ) is $4.91 \times 10^{-7}$, which compares very well to the value of $4.78 \times 10^{-7}$ predicted by Maiorca et al. (2014).

In Fig. 2 the same models of the previous figure are considered but in the terms of $[\mathrm{F} / \mathrm{Fe}]$ versus $[\mathrm{Fe} / \mathrm{H}]$, and we compare our models with the data by Jönsson et al. (2017a) along with Pilachowski \& Pace (2015) data (the stars in Pilachowski \& Pace (2015) are thin disk; see their Sect. 2.1). If we consider both data sets the area spread by the data is well covered by the model predictions. On the other hand, the trend shown by solely the data of Jönsson et al. (2017a), that is, the abundance ratio $[\mathrm{F} / \mathrm{Fe}]$ always increases with $[\mathrm{Fe} / \mathrm{H}]$, is not reproduced by any models presented so far in this paper.

In Fig. 3 we present the model results for the two-infall model in which the W-R star yields by Meynet \& Arnould (2000) for the fluorine are taken into account. As suggested by Renda et al. (2004) and Jönsson et al. (2017a), a possible source for fluorine production are $\mathrm{W}-\mathrm{R}$ stars. These stars might deposit fluorine into the ISM via their strong stellar winds (Meynet \& Arnould 2000; Palacios et al. 2005). Just as in the AGB scenario, the fluorine in 


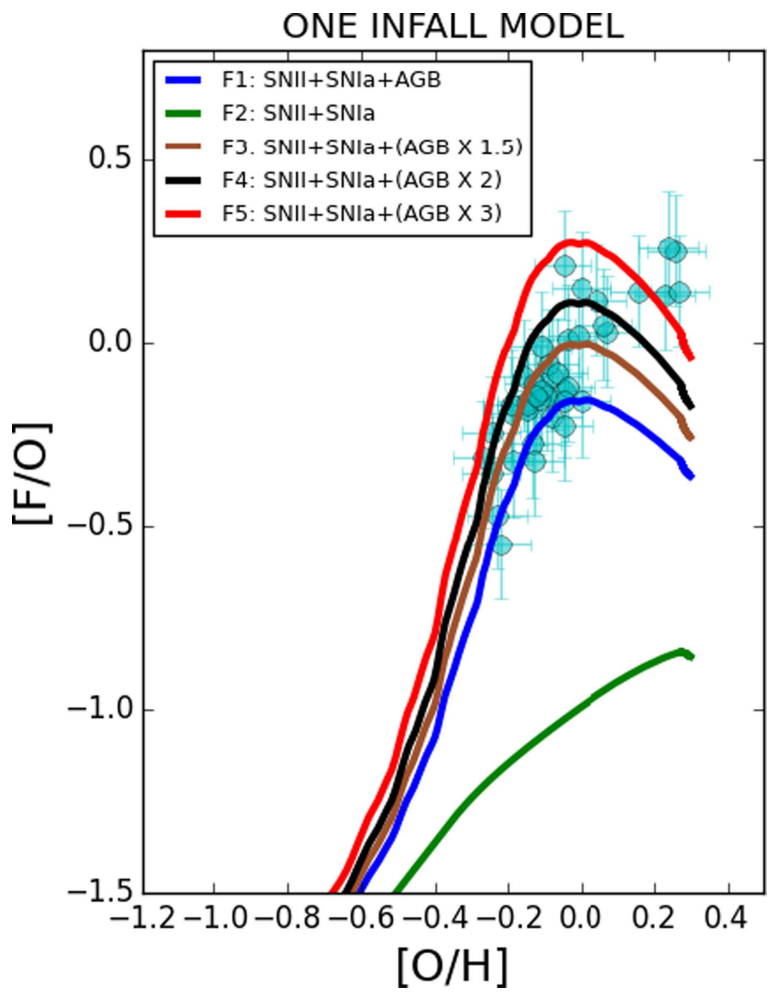

Fig. 6. Abundance ratio $[\mathrm{F} / \mathrm{O}]$ as a function of $[\mathrm{O} / \mathrm{H}]$ in the solar neighborhood for the one-infall chemical evolution model adopting various nucleosynthesis prescriptions for the fluorine production. The model lines are those described in Fig. 1. Observational data of Jönsson et al. (2017a) are indicated with cyan circles, whereas the data taken by Pilachowski \& Pace (2015) are presented with gray pentagons.

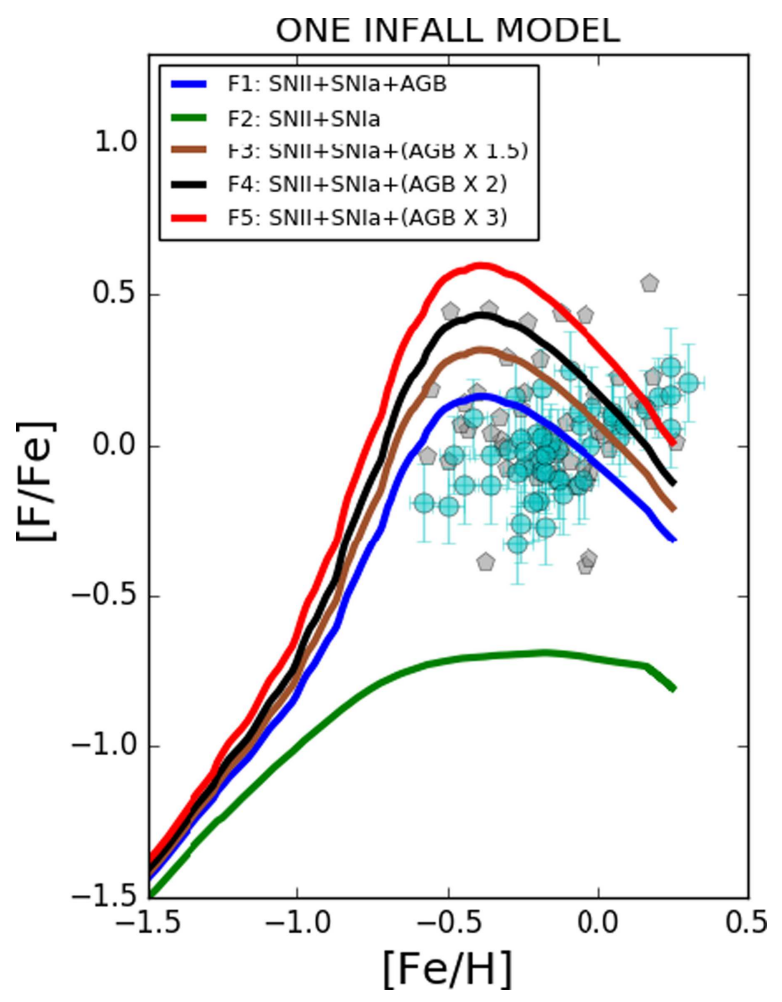

Fig. 7. Abundance ratio $[\mathrm{F} / \mathrm{Fe}]$ as a function of $[\mathrm{Fe} / \mathrm{H}]$ in the solar neighborhood for the one-infall chemical evolution model. The model lines are those described in Fig. 6. Observational data of Jönsson et al. (2017a) are indicated with cyan circles, whereas the data taken by Pilachowski \& Pace (2015) are presented with gray pentagons.
Table 2. Solar values expressed in mass fraction of fluorine predicted the models reported in Table 1 where different channels for the fluorine production are considered.

\begin{tabular}{ccc}
\hline \hline $\begin{array}{c}\text { Fluorine solar values } \\
\left(X_{\mathrm{F}, \odot}\right)\end{array}$ & \multicolumn{2}{c}{ Maiorca et al. $(2014): 4.78 \times 10^{-7}$} \\
2 infall model & 1 infall model \\
\hline F1 & $3.07 \times 10^{-7}$ & $3.03 \times 10^{-7}$ \\
F2 & $9.51 \times 10^{-8}$ & $9.15 \times 10^{-8}$ \\
F3 & $3.99 \times 10^{-7}$ & $3.95 \times 10^{-7}$ \\
F4 & $4.91 \times 10^{-7}$ & $4.87 \times 10^{-7}$ \\
F5 & $6.74 \times 10^{-7}$ & $6.71 \times 10^{-7}$ \\
F6 & $3.23 \times 10^{-7}$ & $3.10 \times 10^{-7}$ \\
F7 & $5.79 \times 10^{-7}$ & $5.64 \times 10^{-7}$ \\
F8 & $8.58 \times 10^{-7}$ & $8.30 \times 10^{-7}$ \\
F9 & $7.62 \times 10^{-7}$ & $7.48 \times 10^{-7}$ \\
\hline
\end{tabular}

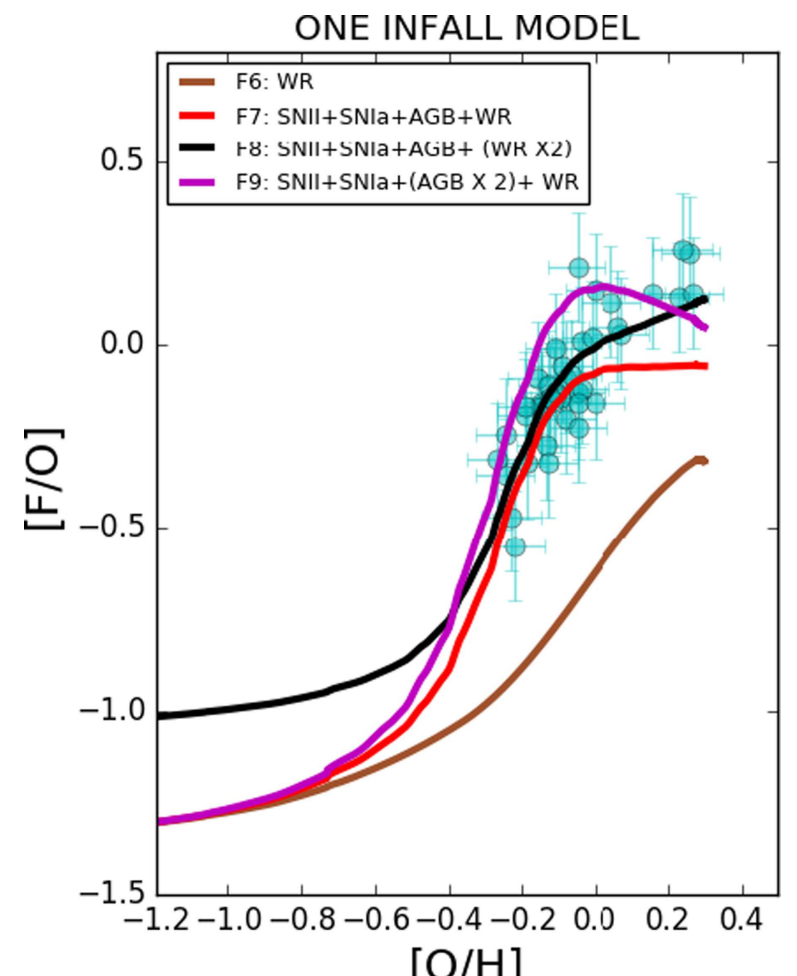

Fig. 8. Abundance ratio $[\mathrm{F} / \mathrm{O}]$ as a function of $[\mathrm{O} / \mathrm{H}]$ in the solar neighborhood for the one-infall chemical evolution model adopting various prescriptions for the channels of fluorine production. The model F6, in which only the contribution for fluorine production is given by W-R stars, is indicated with the brown line. Model F7, in which we adopted the Romano et al. (2010) yield including W-R stars by Meynet \& Arnould (2000), is shown with the red line. Model F8 (the same as model F7, but with the W-R fluorine yields multiplied by 2; see Table 1) is shown with the black line. Finally, models F9 (the same as model F7, but with the AGB fluorine yields multiplied by 2; see Table 1) is labeled with the magenta line. Observational data of Jönsson et al. (2017a) are indicated with cyan circles.

$\mathrm{W}-\mathrm{R}$ winds is produced in reactions starting from ${ }^{14} \mathrm{~N}$, including $\alpha$, neutron, and proton captures. Owing to the strong metallicity dependence of the winds of these stars, a possible fluorine production through this channel is expected to start first at slightly subsolar metallicities and then increase for higher metallicities (Renda et al. 2004).

In Fig. 3 we note that model F7, which considers the Romano et al. (2010) yield prescriptions for oxygen, coupled with W-R 
contribution for the fluorine production, are able to increase the $[\mathrm{F} / \mathrm{O}]$ versus $[\mathrm{O} / \mathrm{H}]$ relation for over-solar values of $[\mathrm{O} / \mathrm{H}]$ in comparison with the reference model $\mathrm{F} 1$. This leads to a better agreement with the data of Jönsson et al. (2017a), but the slope of the observed secondary behavior is not well reproduced and the model shows lower $[\mathrm{F} / \mathrm{O}]$ values at larger $[\mathrm{O} / \mathrm{H}]$. The predicted solar mass fraction for fluorine by the model F7 is $5.79 \times 10^{-7}$, therefore still in perfect agreement with Maiorca et al. (2014) value.

In Fig. 3 we also draw the model F8 (see Table 1 for model yield detail) where we tested the metal dependent W-R yield by Meynet \& Arnould (2000) for fluorine multiplied by hand by a factor of 2 coupled with the Romano et al. (2010) yield. This model is capable of perfectly reproducing the observed data. The predicted solar mass fraction for fluorine by the model F8 is $8.58 \times 10^{-7}$ (see Table 2), which is definitely a higher value than that predicted by Maiorca et al. (2014), but still within a factor of 2 .

In summary, from Table 2 we see that the solar fluorine abundance value reached by the model F7 is in agreement with the Maiorca et al. (2014) value, whereas the model F8 (W-R fluorine yields multiplied by a factor of 2) shows, as expected, a higher value but within a factor of 2 .

We also show the effects of considering fluorine as produced only by W-R stars (model F6). We note that W-R stars contribute to increasing the fluorine production in the whole range of $[\mathrm{O} / \mathrm{H}]$. The main feature of model F6 is that it shows the secondary behavior slope observed in the Jönsson et al. (2017a) stellar sample for $[\mathrm{O} / \mathrm{H}]$ over-solar values. Concerning the $[\mathrm{F} / \mathrm{Fe}]$ versus $[\mathrm{Fe} / \mathrm{H}]$ abundance ratios, Fig. 4 reports the same models described above and shown in Fig. 3.

We see that models including the W-R contribution for the fluorine provide a better fit to the observed data set of Jönsson et al. (2017a) and Pilachowski \& Pace (2015) compared to the models presented in Fig. 2 without this contribution.

We conclude this subsection by focusing on the two-infall model results and present in Fig. 5 the effects of varying AGB fluorine yields by hand in the presence of the W-R fluorine contribution. In model F9 (see Table 1) the AGB yields are multiplied by a factor of 2 along with W-R yields by Meynet \& Arnould (2000). In this way we have a better agreement with data compared to reference model F1.

Without any modification of W-R fluorine yields we find a slight decreasing trend in the $[\mathrm{F} / \mathrm{O}]$ versus $[\mathrm{O} / \mathrm{H}]$ relation for over-solar $[\mathrm{O} / \mathrm{H}]$ values but still in agreement with Jönsson et al. (2017a) data. Moreover the predicted fluorine solar mass fraction is $7.62 \times 10^{-7}$, therefore in better agreement with the Maiorca et al. (2014) value.

\subsection{One-infall model results}

In this subsection we present the results of chemical evolution models for the thin disk of the Galaxy in the solar neighborhood (the one infall model). Figure 6 shows models F1, F2, F3, F4, and F5 (see Table 1 for model detail). In this figure a smoother chemical evolution is evident compared to the two-infall case. This is due to the absence of any gap in the star formation history, which is a peculiar feature of the two-infall (during the transition between the halo-thick disk phase) and thin disk models.

As anticipated in Sect. 4.1, the one-infall model results are able to reproduce the Jönsson et al. (2017a) abundance ratios better. The same models of Fig. 6 are shown in terms of $[\mathrm{F} / \mathrm{Fe}]$ versus $[\mathrm{Fe} / \mathrm{H}]$ in Fig. 7.

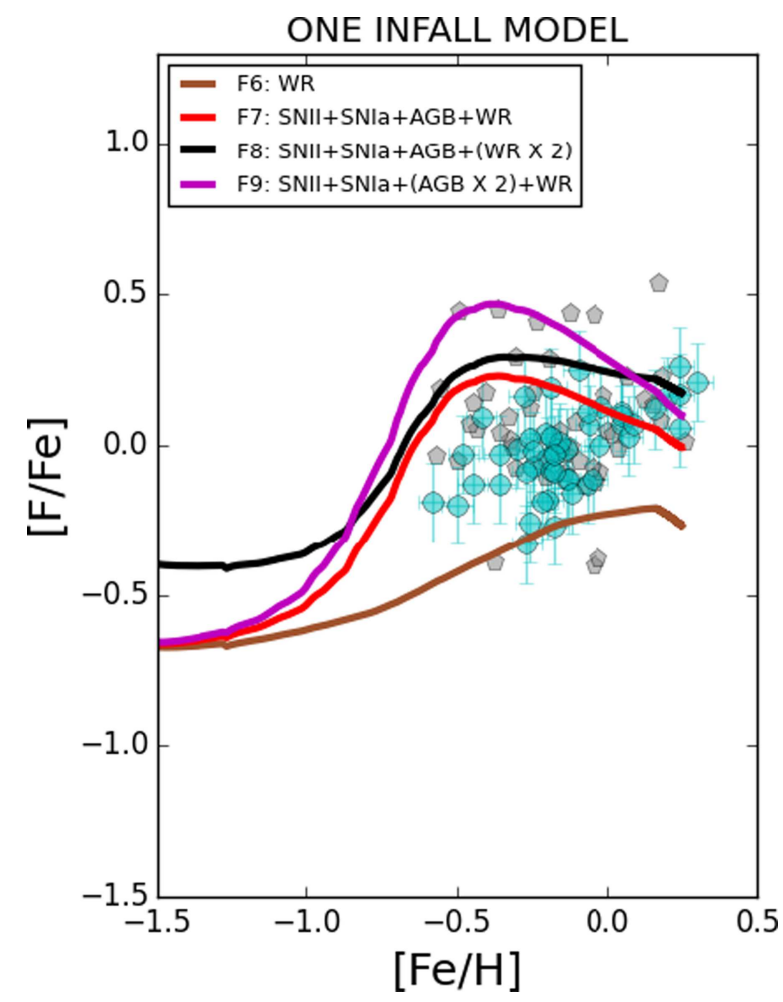

Fig. 9. Abundance ratio $[\mathrm{F} / \mathrm{Fe}]$ as a function of $[\mathrm{Fe} / \mathrm{H}]$ in the solar neighborhood for models F6-F7-F8-F9 applied to the one-infall chemical evolution model. The model lines are the same as Fig. 8. Observational data of Jönsson et al. (2017a) are indicated with cyan circles, whereas the data taken by Pilachowski \& Pace (2015) are presented with gray pentagons.

In Fig. 7 if we consider both data sets, the area spread by the data is well covered by model predictions. On the other hand, the increase of $[\mathrm{F} / \mathrm{Fe}]$ for higher values of $[\mathrm{Fe} / \mathrm{H}]$ shown by the data of Jönsson et al. (2017a) alone, is not reproduced by any model. Moreover, as can be seen in Table 2, the solar values predicted by the one-infall model are not that different from those predicted by the two-infall model, although the former are slightly smaller.

In Fig. 8 we show the effects on the relations [F/O] versus $[\mathrm{O} / \mathrm{H}]$ of including the contribution from the W-R stars to the fluorine production on the one-infall chemical evolution model for the thin disk in terms of $[\mathrm{F} / \mathrm{O}]$ versus $[\mathrm{O} / \mathrm{H}]$ abundance ratios. The model that best fits the data is model F8, which perfectly reproduces the change of slope of the data. In fact, the predicted knee by this model is located around the solar value of $[\mathrm{O} / \mathrm{H}]$, in agreement with the data.

As for the two-infall case, the model F8 shows a higher solar value for the fluorine compared with the Maiorca et al. (2014) value (see Table 2).

Moreover, in Fig. 8 we show the model F9 applied to the oneinfall chemical evolution, in which we multiplied the AGB yields for fluorine along with the W-R yield of Meynet \& Arnould (2000) by a factor of 2. Also, in this case, the model F9 applied to the one-infall chemical evolution model leads to a better fit of the data compared with when we considered the model F9 for the two-infall model. Even if this model predicts a decreasing trend for $[\mathrm{F} / \mathrm{O}]$ versus $[\mathrm{O} / \mathrm{H}]$ for over-solar $[\mathrm{O} / \mathrm{H}]$ values, the model line is within the observed error bars. As reported in Table 2 this model predicts a fluorine solar value that is in better agreement with the Maiorca et al. (2014) value compared to the model F8.

In Fig. 9 models with W-R fluorine contribution are reported for the $[\mathrm{F} / \mathrm{Fe}]$ versus $[\mathrm{Fe} / \mathrm{H}]$ relation. Again, if we consider 

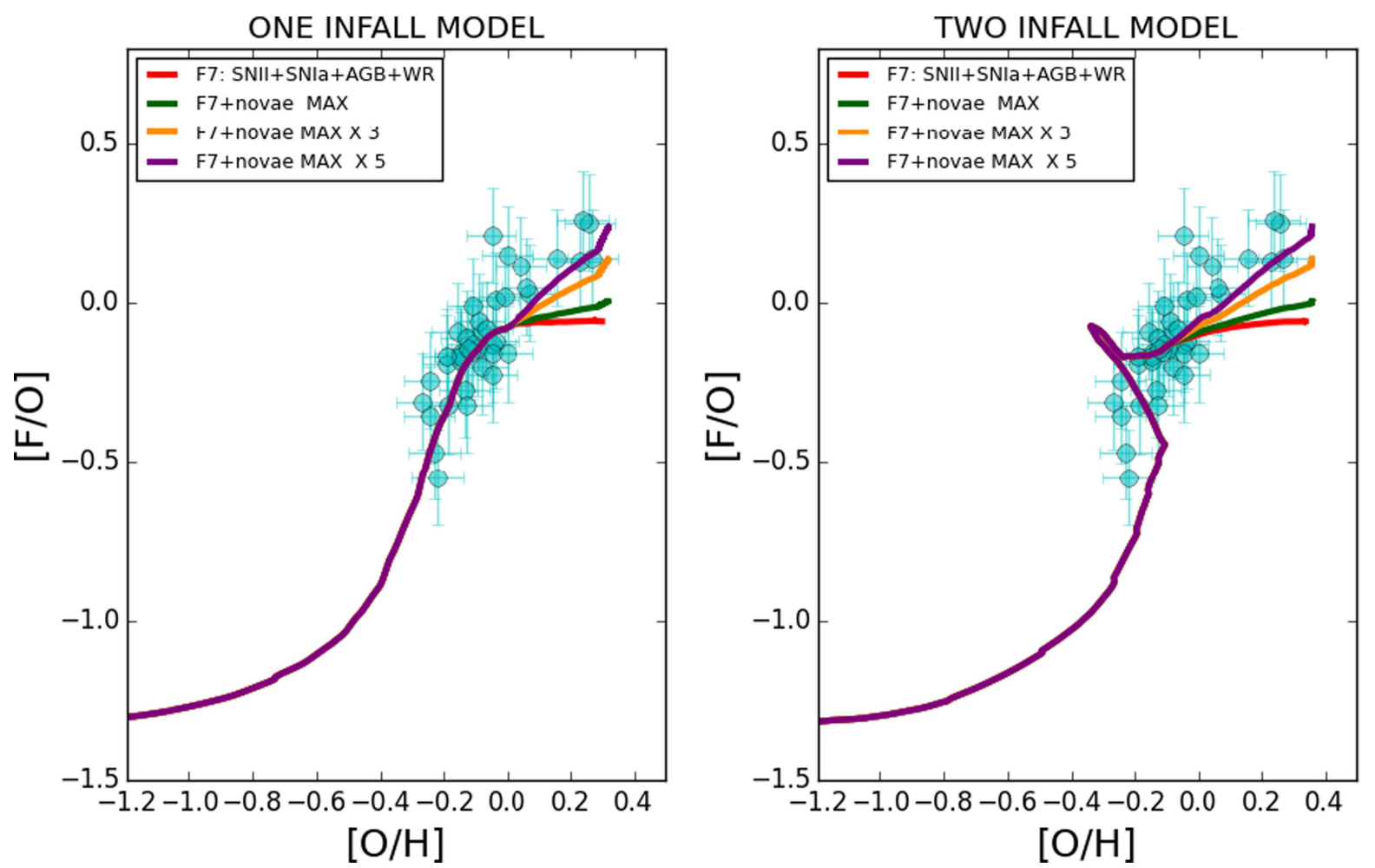

Fig. 10. Abundance ratio $[\mathrm{F} / \mathrm{O}]$ as a function of $[\mathrm{O} / \mathrm{H}]$ in the solar neighborhood for the one-infall (left panel) and two-infall (right panel) chemical evolution models taking into account the effects of the novae. We consider for fluorine the maximum yield by José \& Hernanz (1998; model ONe7) related to ONe white dwarf with masses of $1.35 M_{\odot}$. The blue line indicates the reference F7 model. The model with the "maximum" F novae yield is labeled with the pink line. Models with "maximum" F nova yield multiplied by factor of 5 and 10 are shown with orange and purple lines, respectively.

Pilachowski \& Pace (2015) and Jönsson et al. (2017a) the best model is the F8. However this model it is not able to reproduce the trend shown by the data by Jönsson et al. (2017a). In conclusion, as shown for the two-infall case, we confirm the following results. First, increasing the AGB fluorine yields by hand leads to a better fit of $[\mathrm{F} / \mathrm{O}]$ versus $[\mathrm{O} / \mathrm{H}]$ abundance ratios for subsolar values of $[\mathrm{O} / \mathrm{H}]$, but the secondary behavior at higher $[\mathrm{O} / \mathrm{H}]$ is not reproduced, thereby adopting the nucleosynthesis yields of model 15 of Romano et al. (2010). Second, models that take into account W-R metallicity dependent yields for fluorine by Meynet $\&$ Arnould (2000) are capable of better fitting the observed data. Third, F8 and F9 are the best models.

\section{Testing the novae as possible sources for $F$ production}

In the last section we focus on the effects of nova nucleosynthesis on the fluorine production. In principle this source could help to reproduce the secondary behavior of fluorine observed by Jönsson et al. (2017a) in the $[\mathrm{F} / \mathrm{O}]$ versus $[\mathrm{O} / \mathrm{H}]$ at high $[\mathrm{O} / \mathrm{H}]$ values.

We tested that the inclusion of the nova yields by José \& Hernanz (1998) on the model F7 (see Table 1) has a negligible effect on the chemical evolution of fluorine. In any case, as an exercise, we present in Fig. 10 the model with the maximum fluorine yield by novae, showing the model $\mathrm{F} 7$ in the $[\mathrm{F} / \mathrm{O}]$ versus $[\mathrm{O} / \mathrm{H}]$ relation, including $\mathrm{F}$ produced by novae originated in $\mathrm{ONe}$ white dwarf (W-D) of $1.35 M_{\odot}$ (model ONe7 of José \& Hernanz 1998). Although this W-D model leads the maximum production of $\mathrm{F}$ in novae, we are aware that these objects are extremely rare in nature. In the same figure we present model results in which the fluorine nova yields are multiplied by factors of 3 and 5 , respectively.

It is quite interesting to note that in this way we are able to trace perfectly the secondary trend at high $[\mathrm{O} / \mathrm{H}]$ values. However, we note that nova yields are still very uncertain. It is worth noting that Li synthesized in a nova outburst as inferred from recent observations (Tajitsu et al. 2015; Izzo et al. 2015) exceeds by far that expected from the theoretical models of José \& Hernanz (1998). This fact suggests that there is also room for a significant revision of $F$ yield from novae.

\section{Conclusions}

In this article we studied in detail the effects of various nucleosynthesis prescriptions for the fluorine production on the chemical evolution models for the solar neighborhood with the aim of reproducing the observational data by Jönsson et al. (2017a). Our main conclusions can be summarized as follows:

- The role played by W-R stars in the fluorine production seems to be essential to reproduce the new observed ratios in the solar neighborhood $[\mathrm{F} / \mathrm{O}]$ versus $[\mathrm{O} / \mathrm{H}]$ by Jönsson et al. (2017a). This confirms previous suggestions by Renda et al. (2004).

- We obtain a better agreement with the observed fluorine abundances when we consider the one-infall chemical evolution model, relative only to the thin disk.

- The best one-infall model reproducing the observed abundance ratios $[\mathrm{F} / \mathrm{O}]$ versus $[\mathrm{O} / \mathrm{H}]$ for the thin disk requires the nucleosynthesis prescriptions of the model 15 of 
Romano et al. (2010) along with the W-R fluorine yields by Meynet \& Arnould (2000) multiplied by a factor of 2. On the other hand, this model predicts a solar value that is higher than that of Maiorca et al. (2014).

- Considering the AGB yields by Karakas (2010) multiplied by a factor of 2 along with the Meynet \& Arnould (2000) $\mathrm{W}-\mathrm{R}$ yields leads to a good fit of the $[\mathrm{F} / \mathrm{O}]$ versus $[\mathrm{O} / \mathrm{H}]$ data and predicts a solar value in agreement with Maiorca et al. (2014) value.

- Concerning the $[\mathrm{F} / \mathrm{Fe}]$ versus $[\mathrm{Fe} / \mathrm{H}]$ relation, the models presented here are in agreement with the collection of data composed by Jönsson et al. (2017a) and Pilachowski \& Pace (2015). The data by Jönsson et al. (2017a) show that [F/Fe] abundances increase with $[\mathrm{Fe} / \mathrm{H}]$. This trend is not found by our models for $[\mathrm{Fe} / \mathrm{H}]$ values higher than -0.3 dex. We want to emphasize that there are still huge uncertainties concerning the nucleosynthesis of $\mathrm{F}$ and this could be the reason for the discrepancy.

- More detailed data for fluorine in the solar neighborhood are required at low metallicities, i.e., for $[\mathrm{O} / \mathrm{H}]$ values lower than -0.4 dex, to confirm the importance of W-R stars in the fluorine production, as we conclude in this paper. In fact, we predict that the inclusion of the fluorine produced by W-R stars would affect the $[\mathrm{F} / \mathrm{O}]$ versus $[\mathrm{O} / \mathrm{H}]$ ratio also at low $[\mathrm{O} / \mathrm{H}]$ values, leading to a roughly flat $[\mathrm{F} / \mathrm{O}]$ ratio for $[\mathrm{O} / \mathrm{H}]$ smaller than -0.5 dex.

- We also show that the novae, even if their yields are still uncertain could help to better reproduce the secondary behavior of $\mathrm{F}$ in the $[\mathrm{F} / \mathrm{O}]$ versus $[\mathrm{O} / \mathrm{H}]$ relation in presence of W-R stars fluorine contribution.

In conclusion, the inclusion of the fluorine production by $\mathrm{W}-\mathrm{R}$ stars seems to be essential to reproduce the new observed ratio $[\mathrm{F} / \mathrm{O}]$ versus $[\mathrm{O} / \mathrm{H}]$ in the solar neighborhood. Moreover, the inclusion of novae helps to reproduce the observed fluorine secondary behavior substantially.

Acknowledgements. We thank the anonymous referee for the suggestions that improved the paper. E. Spitoni and F. Matteucci thank the financial support by FRA2016 - University of Trieste. N. Ryde acknowledges support from the Swedish Research Council, VR (project number 621-2014-5640), Funds the Royal Physiographic Society of Lund (Stiftelsen Walter Gyllenbergs fond and Märta och Erik Holmbergs donation), and from the project grant "The New Milky" from the Knut and Alice Wallenberg foundation. H. Jönsson acknowledges the support by the Lars Hierta Memorial Foundation, Helge Ax:son Johnsons stiftelse, and Stiftelsen Olle Engkvist Byggmästare.

\section{References}

Asplund, M., Grevesse, N., Sauval, A. J., \& Scott, P. 2009, ARA\&A, 47, 481 Brusadin, G., Matteucci, F., \& Romano D. 2013, A\&A, 554, A135

Cescutti, G., Matteucci, F., Caffau, E., \& François, P. 2012, A\&A, 540, A33 Chiappini, C., Matteucci, F., \& Gratton, R. 1997, ApJ, 477, 765

Chiappini, C., Matteucci, F., \& Romano, D. 2001, ApJ, 554, 1044

Cristallo, S., Di Leva, A., Imbriani, G., et al. 2014, A\&A, 570, A46

Ekström, S., Meynet, G., Chiappini, C., Hirschi, R., \& Maeder, A. 2008, A\&A, 489, 685

François, P., Matteucci, F., Cayrel, R., et al. 2004, A\&A, 421, 613

Grisoni, V., Spitoni, E., Matteucci, F., et al. 2017, MNRAS, 472, 3637

Hayden, M. R., Bovy, J., Holtzman, J. A., et al. 2015, ApJ, 808, 132

He, J. J., Lombardo, I., Dell'Aquila, D., et al. 2018, Chin. Phys. C, 42, 015001

Hirschi, R. 2005, in From Lithium to Uranium: Elemental Tracers of Early Cosmic Evolution, eds. V. Hill, P. François, \& F. Primas (Dordrecht: Kluwer), Proc. IAU Symp., 228, 331

Hirschi, R. 2007, A\&A, 461, 571

Indelicato, I., La Cognata, M., Spitaleri, C., et al. 2017, ApJ, 845, 19

Iwamoto, K., Brachwitz, F., Nomoto, K., et al. 1999, ApJS, 125, 439

Izzo, L., Della Valle, M., Mason, E., et al. 2015, ApJ, 808, L14

Jönsson, H., Ryde, N., Spitoni, E., et al. 2017a, ApJ, 835, 50

Jönsson, H., Ryde, N., Nordlander, T., et al. 2017b, A\&A, 598, A100

José, J., \& Hernanz, M. 1998, ApJ, 494, 680

Karakas, A. I. 2010, MNRAS, 403, 1413

Kennicutt, R. C., Jr. 1989, ApJ, 344, 685

Kennicutt, R. C., Jr. 1998, ApJ, 498, 541

Kobayashi, C., Umeda, H., Nomoto, K., Tominaga, N., \& Ohkubo, T. 2006, ApJ, 653,1145

Kobayashi, C., Izutani, N., Karakas, A. I., et al. 2011a, ApJ, 739, L57

Kobayashi, C., Karakas, A. I., \& Umeda, H. 2011b, MNRAS, 414, 3231

Kuijken, K., \& Gilmore, G. 1991, ApJ, 367, L9

Maas, Z. G., Pilachowski, C. A., \& Cescutti, G. 2017, ApJ, 841, 108

Maiorca, E., Uitenbroek, H., Uttenthaler, S., et al. 2014, ApJ, 788, 149

Martin, C. L., \& Kennicutt, R. C., Jr. 2001, ApJ, 555, 301

Matteucci, F., \& François, P. 1989, MNRAS, 239, 885

Meynet, G., \& Arnould, M. 1993, in Nuclei in the Cosmos II, eds. F. Kaappeler, K. Wisshak (Bristol: IOP), 503

Meynet, G., \& Arnould, M. 2000, A\&A, 355, 176

Meynet, G., \& Maeder, A. 2002, A\&A, 390, 561

Micali, A., Matteucci, F., \& Romano, D. 2013, MNRAS, 436, 1648

Mikolaitis, S., de Laverny, P., Recio-Blanco, A. et al. 2017, A\&A, 600, A22

Palacios, A., Arnould, M., \& Meynet, G. 2005, A\&A, 443, 243

Pilachowski, C. A., \& Pace, C. 2015, AJ, 150, 66

Recio-Blanco, A., et al. 2014, A\&A, 567, A5

Renda, A., Fenner, Y., Gibson, B. K., et al. 2004, MNRAS, 354, 575

Rojas-Arriagada, A., Recio-Blanco, A., de Laverny, P., et al. 2017, A\&A, 601, A140

Romano, D., Karakas, A. I., Tosi, M., \& Matteucci, F. 2010, A\&A, 522, A32

Spitoni, E., Romano, D., Matteucci, F., \& Ciotti, L. 2015, ApJ, 802, 129

Spitoni, E., Vincenzo, F., Matteucci, F., \& Romano, D. 2016, MNRAS, 458, 2541

Tajitsu, A., Sadakane, K., Naito, H., Arai, A., \& Aoki, W. 2015, Nature, 518, 381

Woosley, S. E., \& Haxton, W. C. 1988, Nature, 334, 45

Woosley, S. E., \& Weaver, T. A. 1995, ApJ, 101, 181 